\title{
The CMS Electromagnetic Calorimeter: lessons learned during LHC run 1, overview and future projections
}

\author{
Arabella Martelli* ${ }^{*}$ \\ Physics Department, Università degli Studi di Milano Bicocca, \\ Piazza della Scienza 3, 20156 Milano, Italy \\ E-mail: arabella.martelli@cern.ch
}

\begin{abstract}
The Electromagnetic Calorimeter (ECAL) of the Compact Muon Solenoid (CMS) experiment at the LHC is a hermetic, fine grained, homogeneous calorimeter, comprising 75848 lead tungstate scintillating crystals. We highlight the key role of the ECAL in the discovery and elucidation of the Standard Model Higgs boson during LHC Run I. We discuss, with reference to specific examples from LHC Run I, the challenges of operating a crystal calorimeter at a hadron collider. Particular successes, chiefly in terms of achieving and maintaining the required detector energy resolution in the harsh radiation environment of the LHC, are described. The prospects for LHC Run II (starting in 2015) are discussed, building upon the experience gained from Run I. The high luminosity upgrade of the LHC (HL-LHC) is expected to be operational from about 2025 to 2035 and will provide instantaneous and integrated luminosities of around $5 \times 10^{34} \mathrm{~cm}^{-2} \mathrm{~s}^{-1}$ and $3000 \mathrm{fb}^{-1}$ respectively. We outline the challenges that ECAL will face and motivate the evolution of the detector that is thought to be necessary to maintain its performance throughout LHC and High-Luminosity LHC operation.
\end{abstract}

Technology and Instrumentation in Particle Physics 2014

2-6 June, 2014

Amsterdam, the Netherlands

\footnotetext{
* Speaker.

${ }^{\dagger}$ On behalf of the CMS collaboration.
} 


\section{The ECAL design}

The electromagnetic calorimeter (ECAL) [1] of the CMS experiment at the LHC has been specifically designed to optimise the sensitivity to the $H \rightarrow \gamma \gamma$ decay mode. This decay channel requires excellent diphoton invariant mass resolution, hence precise single photon energy and position determination, and good photon identification capabilities, achieved with fine transverse granularity. These characteristics, complemented by the ECAL wide acceptance, were key ingredients also to study the four electrons final state, of the $H \rightarrow Z Z$ decay channel.

The $75848 \mathrm{PbWO}_{4}$ scintillating crystals, of which the detector is built, are arranged in a quasiprojective geometry in the central barrel (EB) and in the two endcaps (EE), covering the pseudorapidity range $|\eta|<1.48$ and $1.48<|\eta|<3$, respectively. The scintillating light is readout by avalanche photodiodes (APDs) in the EB, and vacuum phototriodes (VPTs) in the EE. The small Moliere radius $(22 \mathrm{~mm})$ and short radiation length $(8.9 \mathrm{~mm})$ result in compact structure and high granularity, while providing excellent energy containment.

Before installation in CMS, the performance of the calorimeter components have been extensively tested on beam, with high energy electrons and under ideal conditions: no magnetic field, no upstream material, fixed impact point. The energy resolution was measured to be well below $1 \%$ at high energies, with an irreducible constant term of about $0.3 \%$ [2].

During data taking in CMS, the residual inter-channel miscalibration, the time-dependent variation of the response, due to radiation damage and environmental instability, as well as the gain spread among the VPTs contribute to the energy resolution. The energy resolution is further degraded for electrons and photons interacting in the tracker material in front of ECAL.

Specific reconstruction algorithms and additional calibration factors are needed to preserve the excellent intrinsic resolution of ECAL.

\section{Energy Estimation}

The ECAL is expected to provide the most accurate estimate of the energy for electrons and photons. Electromagnetic particles generate showers in ECAL, depositing energy over several crystals. Furthermore, energy deposits due to secondary emission in the tracker material, by bremsstrahlung or photon conversions, are spread along the $\phi$ direction in ECAL by the intense magnetic field. In ECAL, the energy estimate is obtained through a dynamic superclustering algorithm which performs a sum over the channels involved in the shower and is particularly flexible along the $\phi$ direction.

The energy $\left(E_{e / \gamma}\right)$ is obtained from the sum of the signals from the crystals in the cluster, as in 2.1

$$
E_{e / \gamma}=G \cdot F_{e / \gamma} \cdot \Sigma_{i}\left[S_{i}(t) \cdot C_{i} \cdot A_{i}\right]
$$

where $A_{i}$ is the channel amplitude in the $i^{t h}$ channel, $S_{i}(t)$ are time-dependent coefficients that equalise the channel response to compensate for transparency losses and $C_{i}$ takes into account the intrinsic spread in light-yield among crystals. The sum is multiplied by the ADC-to-energy conversion factor $(G)$ and by a function that accounts for imperfect clustering and geometry effects $\left(F_{e / \gamma}\right)$. 

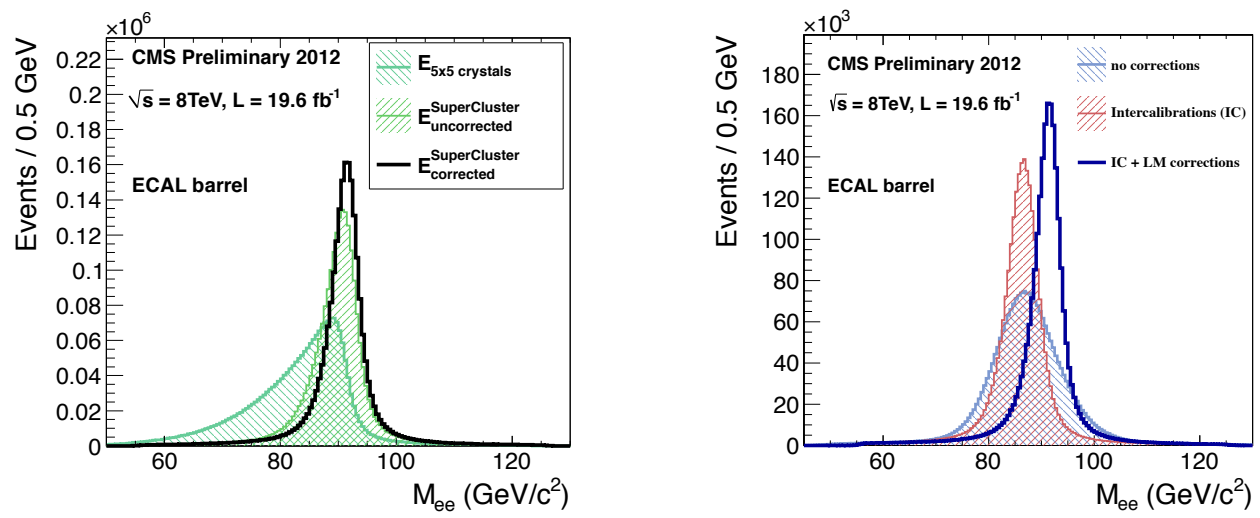

Figure 1: Impact on the invariant mass of $Z \rightarrow e e$ events in EB from: the dynamic superclustering algorithm and energy corrections (on the left), the intercalibration and transparency corrections (on the right).

Figure 1 (left) shows the invariant mass distribution from $Z \rightarrow e e$ events using the simple sum of the 25 crystals around the maximum, the uncorrected energy of the supercluster and the energy of the supercluster corrected by $F_{e / \gamma}$. Figure 1 (right) shows how the invariant mass resolution is improved by applying the intercalibration constants $C_{i}$ and the transparency corrections $S_{i}(t)$.

Given the importance of such correction factors for accurate energy measurement and excellent energy resolution, great care is devoted to the computation and monitoring of such factors.

\section{ECAL operation and monitoring}

\subsection{Time-dependent Response Changes}

Radiation damage is responsible for the loss of transparency in the ECAL crystals, through the creation of crystals defects. In particular, hadron damage to $\mathrm{PbWO}_{4}$ crystals is known to be a major problem at room temperature. It is permanent and cumulative, while transmission losses from $\gamma$ irradiation spontaneously recover. VPT conditioning also contributes to response loss for channels in the endcap region, due to the cumulated charge on the photo-cathode.

The channels response is constantly monitored during operation by means of a laser monitoring system [5], which provides one monitoring point per crystal every 40 minutes, with single point precision better than $0.1 \%$ and long-term instabilities of about $0.2 \%$. In Figure 2, the evolution of the ECAL channels response is shown for different $\eta$ ranges, as measured in 2011-2012, with the laser monitoring system. Losses are of the order of a few percent in the EB and reach $\simeq 30 \%$ in the most forward EE regions used for electron and photon reconstruction $(|\eta| \simeq 2.5)$. The cycles of loss and recovery, mostly due to transmission losses from ionising radiation damage and subsequent annealing, are also visible in the Figure. The effects observed are consistent with radiation hardness tests performed during construction [6].

The monitoring information is used to prepare correction factors $\left(S_{i}(t)\right)$, which are delivered for the prompt reconstruction, within $48 \mathrm{~h}$ from the data taking. The stability of the correction factors is checked using electrons from $\mathrm{W}$ decays (Fig. 2 right). Corrections are also applied at the level-1 trigger, to provide full triggering efficiency. This was done for the endcaps in 2012 and will be extended to the barrel for 2015. In Figure 3 the efficiency curve measured in the endcap for a 

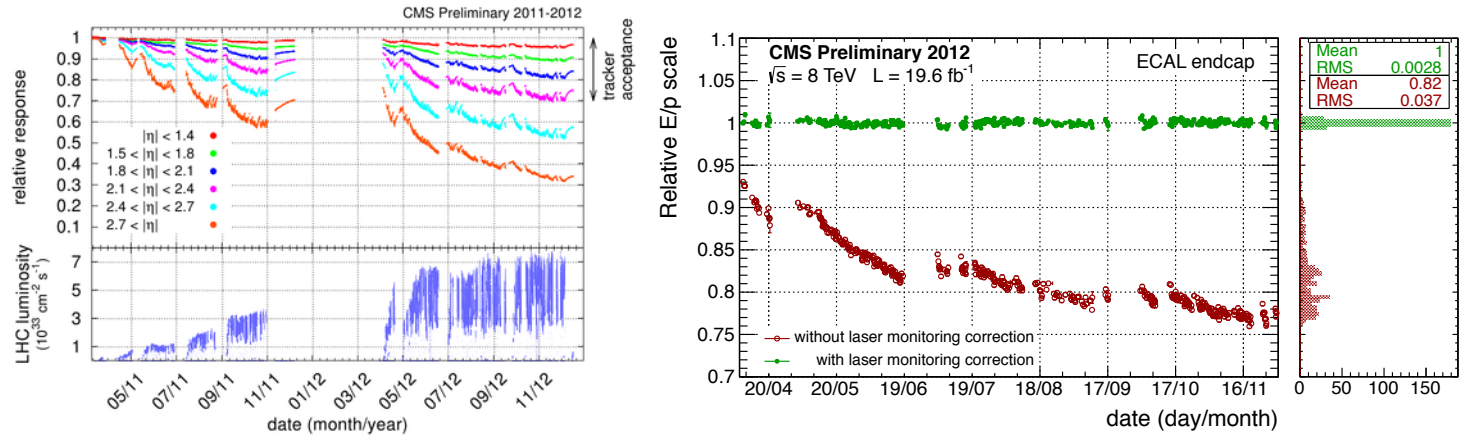

Figure 2: Left: relative response to laser light (440 $\mathrm{nm}$ in 2011 and $447 \mathrm{~nm}$ in 2012) measured by the ECAL laser monitoring system, averaged over all crystals in bins of pseudo-rapidity, for the 2011 and 2012 data taking periods. Right: ratio of electron energy E, measured in the ECAL endcaps, to the electron momentum $\mathrm{p}$, measured in the tracker, before and after correcting for response losses using the laser monitoring system, shown as a function of time for 2012 data.

specific trigger $\left(E_{T}>20 \mathrm{GeV}\right)$ is compared to the one measured in the barrel, where the response loss is much smaller.

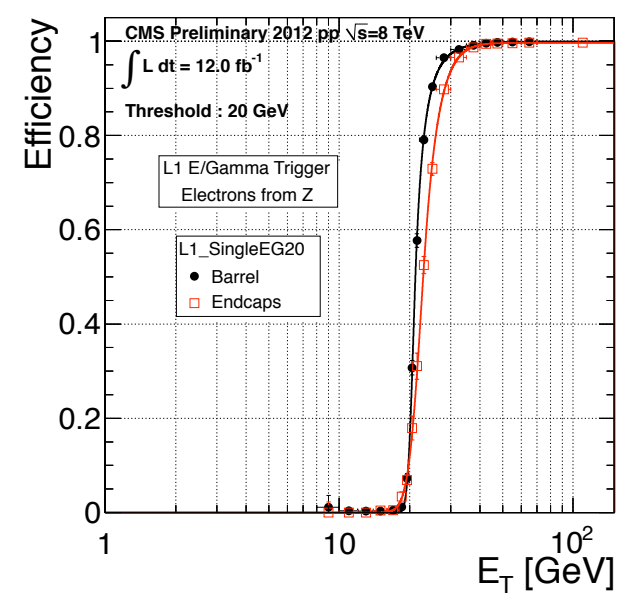

Figure 3: Efficiency curve for the EG20 trigger (transverse energy $E_{T}$ measured in towers of $5 \times 5$ crystals for electrons and photons candidates greater than $20 \mathrm{GeV}$ ) shown as a function of particle energy, for the EB and EE of the CMS ECAL.

\subsection{Inter-crystal calibration}

In ECAL, several methods are used to compute the crystal intercalibration coefficients $\left(C_{i}\right)$. These include the invariance around the beam axis of the energy flow in minimum bias events $(\phi$ symmetry), the $\pi^{0} / \eta$ mass constraint on the energy of the two photons from $\pi^{0} / \eta \rightarrow \gamma \gamma$ decays and the momentum constraint on the energy of isolated electrons from $\mathrm{W}$ decays.

The individual methods are validated with cross-comparison and combined. The precision on the coefficients obtained with 2012 data is shown in Fig. 4 in function of pseudorapidity for EB (left) and EE (right). 

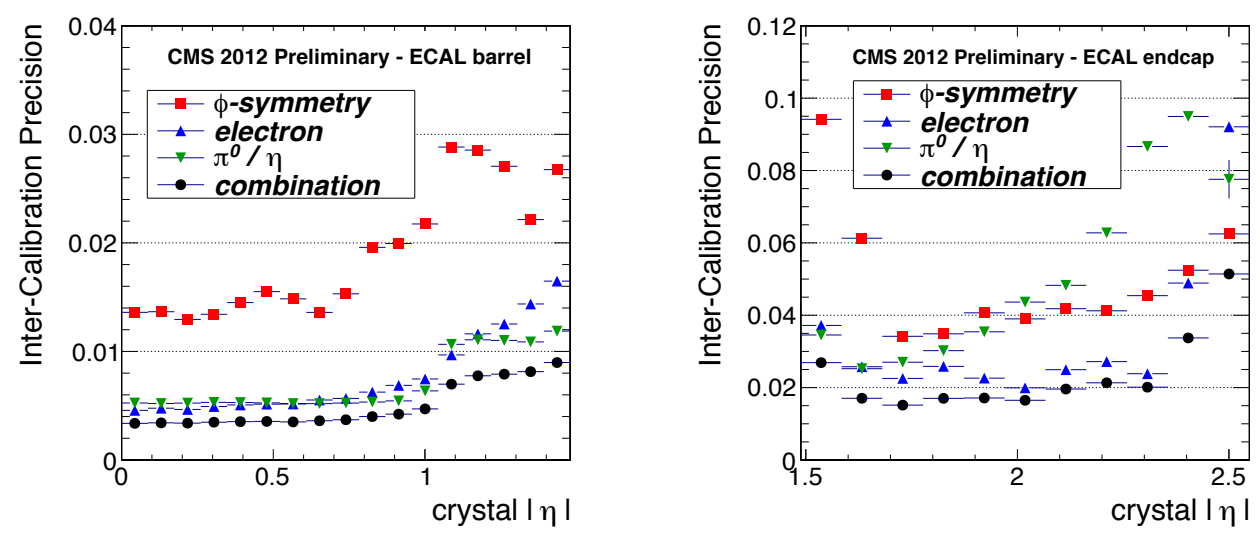

Figure 4: Precision of the various calibration sets used in 2012 in EB (left) and EE (right).

The residual miscalibration of the channel response ensures a contribution to the energy resolution below $0.4 \%$ in the central part of the barrel $(|\eta|<1)$ which is an excellent result. Is is below $1 \%$ overall in EB and at the level of $2 \%$ on average in the endcaps.

\section{Energy Resolution}

The energy resolution is studied with $Z \rightarrow e e$ events. The instrumental contribution to the $Z$ width is extracted from a fit to the invariant mass distribution, modeling the signal by a convolution of a Breit-Wigner and a Crystal-Ball response function. The result is shown as a function of $|\eta|$ in Fig. 5 (left) for data, both prompt (grey) and final (blue) reconstruction, and simulation (red).
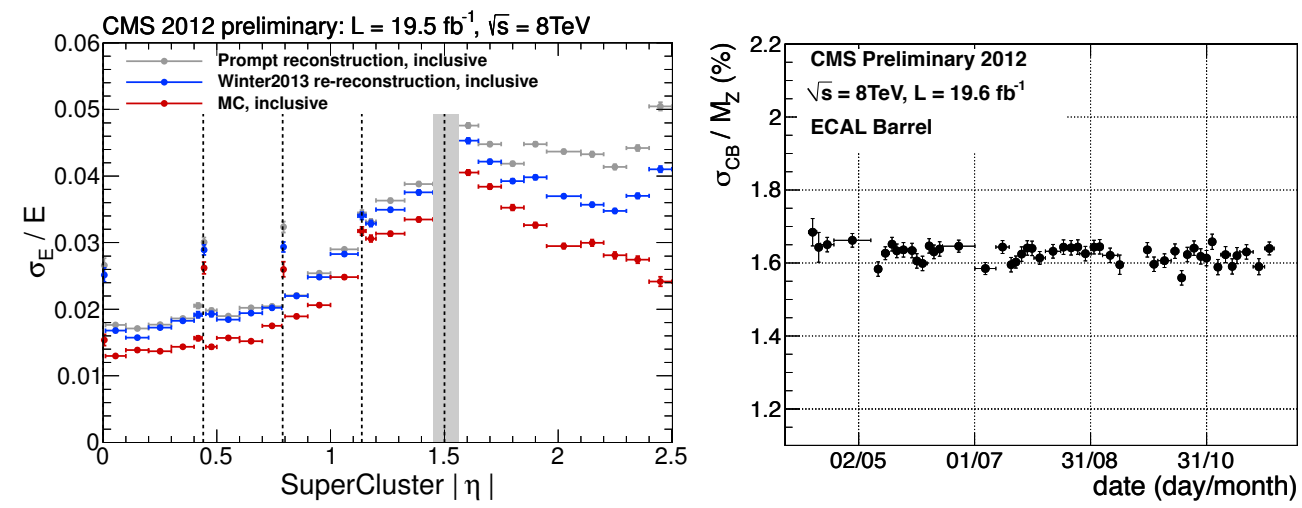

Figure 5: Left: electron energy resolution for the ECAL using $Z \rightarrow e e$ events. The resolution is affected by the material in front of the ECAL and is degraded in the vicinity of cracks between ECAL modules at specific $|\eta|$, shown by the vertical lines in the plot. Right: instrumental invariant mass resolution measured on 2012 data with $Z \rightarrow e e$ events as a function of time.

The prompt reconstruction is already very good, especially in the barrel. With the refined conditions used for the final reconstruction, the resolution significantly improves, especially in the endcaps. Moreover the mass resolution on $Z \rightarrow e e$ data events is shown to be stable at the 1 permil level as a function of time (Fig. 5 right). 
Residual differences are visible between data and simulation (Fig. 5 left), with a clear modulation and trend as a function of $|\eta|$. Reasons for this are the imperfect modelling in the simulation of the material upstream ECAL and of the response changes with time, as measured in data.

Recently a more realistic simulation has been implemented in CMS. Moreover, any residual difference between data and MC is taken into account by adding extra-smearing contributions to the $\mathrm{MC}$ resolution. The tuning of the MC resolution to match the one measured in data is crucial for the $H \rightarrow \gamma \gamma$ analysis.

\section{Photon identification}

The ECAL granularity plays an important role for the photon identification, which is performed through the use of several variables.

The variable $r 9=E_{3 \times 3} / E_{\text {raw }}$, defined as the ratio of the energy in a $3 \times 3$ array of crystals to the energy in the supercluster before algorithmic corrections, allows to classify photons of high or low resolution. According to MC, about $70 \%$ of the photons with $r 9>0.94$ are truly unconverted, while all the photons with $r 9<0.94$ interact upstream ECAL. The good level of agreement between the $r 9$ distributions for data and MC is shown in Figure 6 (left) for photons in EB. Additional calorimetric variables used in the photon ID are the transverse spread of electromagnetic showers, crucial for the $\pi^{0} / \eta$ separation, and the isolation, used to separate photons from electromagnetic deposits associated with jet fragments.

The photon identification efficiency is estimated from simulation (Fig. 6 right) to be better than $90 \%$ in the barrel, decreasing to about $60 \%$ at $|\eta|=2.5$. The background to the selected $H \rightarrow \gamma \gamma$ sample [7] is largely irreducible, from prompt photon production. About $30 \%$ of the background contains one or more misidentified photons, mainly from $\gamma+$ jet production. The MC background composition and shape are not used in the search of the $H \rightarrow \gamma \gamma$, but only to tune photon identification selections.
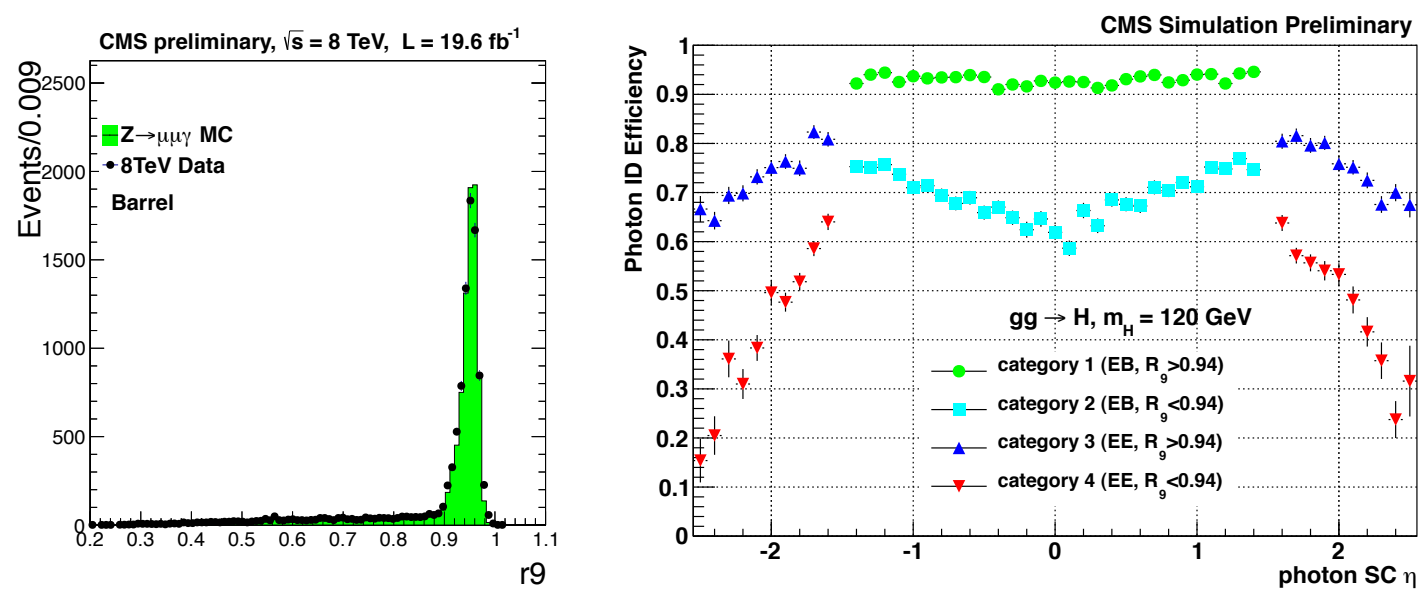

Figure 6: Left: distribution of $r 9$ in data and MC for photons in $Z \rightarrow \mu \mu \gamma$ decays. Right: photon identification efficiency as a function of $\eta$ for high and low $r 9$ candidates. 


\section{Position reconstruction}

An accurate reconstruction of the photon direction is important to obtain good $\gamma \gamma$ mass resolution, as this depends on the accuracy in measuring the opening angle between the two photons. This is determined from the position of the showers in ECAL and the location of the interaction vertex. The vertex assignment uses multivariate techniques and relies on the kinematic properties of the tracks associated with that vertex and on their correlation with the diphoton kinematics as measured in ECAL. MC studies show that the efficiency of correct assignment (within $1 \mathrm{~cm}$ from the true vertex) is close to $100 \%$, for transverse momentum of the $\gamma \gamma$ system above $100 \mathrm{GeV}$.

\section{Implications for the $H \rightarrow \gamma \gamma$ hunt}

The $H \rightarrow \gamma \gamma$ search profits from a multivariate analysis used to categorise diphoton events, based on event topologies and kinematics, to exploit the dependence of the resolution on the properties of the reconstructed photons and on their position in ECAL.

In each category, the background is estimated from data with a parametric fit to the $\gamma \gamma$ candidates invariant mass spectrum on a range wider than the signal search window. The signal model is derived from the MC simulation of the $H \rightarrow \gamma \gamma$ decay. In order to precisely simulate the ECAL response to photons, the constant term of the energy resolution in $\mathrm{MC}$ is tuned in different $\eta$ regions of ECAL and for different electromagnetic shower shapes $r 9$, to match the resolution observed in the $Z \rightarrow e e$ decay, where the electrons are reconstructed as photons except for vertex location.

The shape of the expected signal is shown in Figure 7, for the inclusive sample of $H \rightarrow \gamma \gamma$ categories. There has been a significant improvement over time, due to progress in calibration and clustering. The good precision achieved with prompt reconstruction and $7 \mathrm{TeV}$ data, improves moving to the final reconstruction and the use of refined calibrations and corrections. The results are further improved moving to $8 \mathrm{TeV}$ data, in spite of the worsening of the LHC running condition. This is a result achieved with an high level of understanding of the detector effects.

These efforts were fundamental for the Higgs discovery and high quality measurement in the $H \rightarrow \gamma \gamma$. The latest published CMS result [8] presents a clean signal, with local significance of just over $3 \sigma$, and high precision mass measurement (Fig. 8)

\section{Perspectives for the future}

The CMS electromagnetic calorimeter has successfully operated during the LHC run 1, meeting the stringent design requirements, driven by the hunt for the SM $H \rightarrow \gamma \gamma$. The good understanding and control of the detector effects delivered the conditions for the $H \rightarrow \gamma \gamma$ observation with high sensitivity. The excellent performance of the ECAL barrel, with excellent resolution and photon ID capabilities, was fundamental in driving the sensitivity to the $H \rightarrow \gamma \gamma$ search. The endcap part, where the acceptance to $H \rightarrow \gamma \gamma$ photons is lower, played a limited role in this search, due to the lower identification efficiency and non optimal resolution. Margin for improvements are already identified in the workflow described in the previous sections. A fine-tuning of the calibration and time-dependent corrections, as well as a more accurate description of the ECAL upstream material, together with possible enhanced reconstruction algorithms are expected to further improve the 

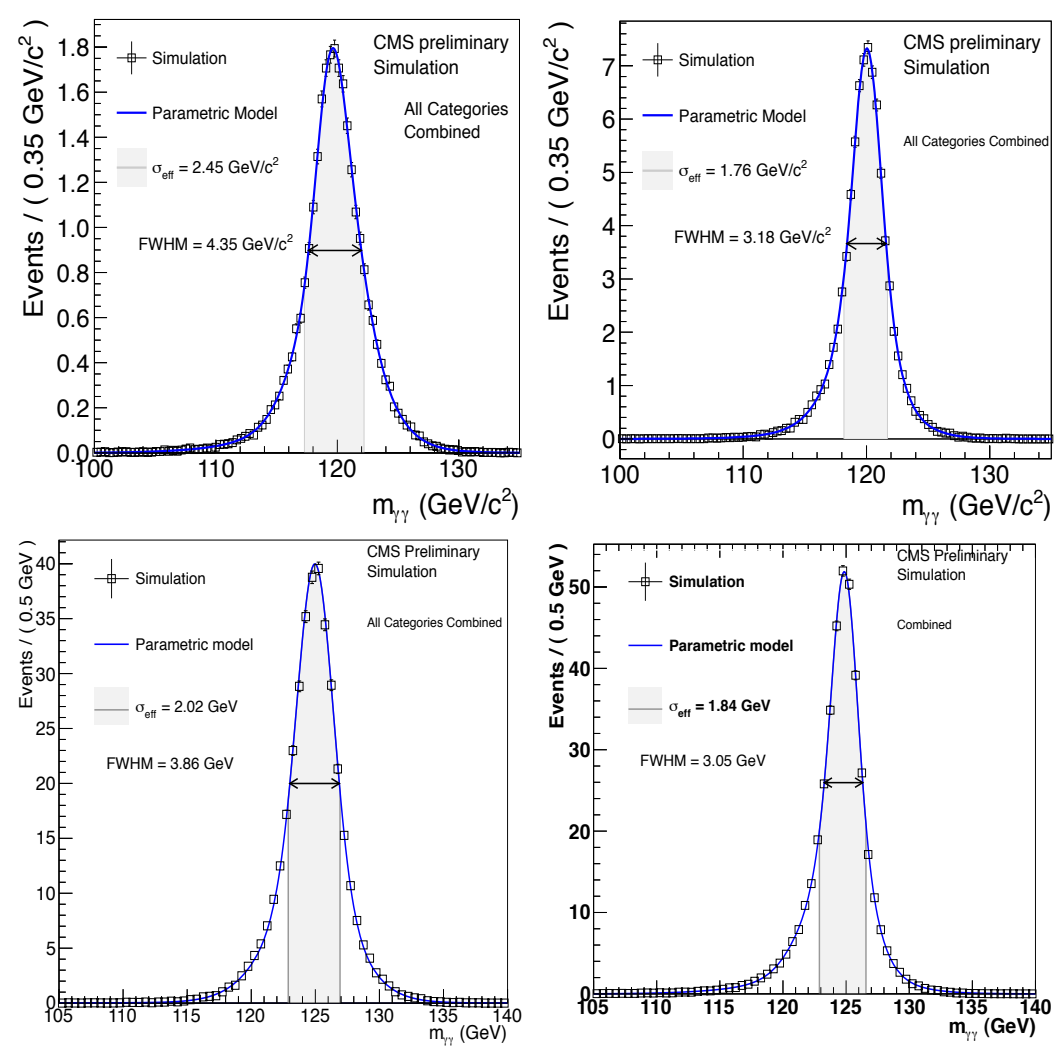

Figure 7: Evolution, from 2011 to 2013, of the simulated $H \rightarrow \gamma \gamma$ mass resolution, using energy resolution from data. Top left: prompt reconstruction, $7 \mathrm{TeV}$ (LP 2011). Top right: final reconstruction, $7 \mathrm{TeV}$ (ICHEP 2012). Bottom left: prompt reconstruction, $8 \mathrm{TeV}$ (MORIOND 2013). Bottom right: final reconstruction, 8 $\mathrm{TeV}$ (Legacy paper 2014).
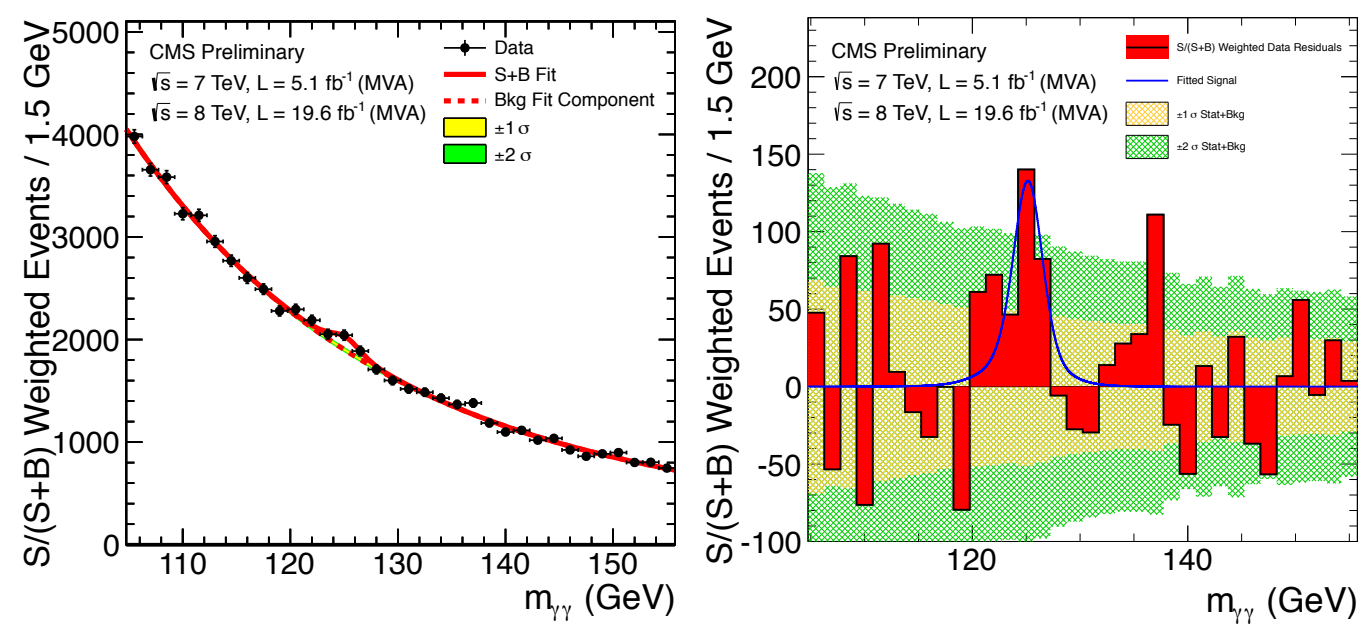

Figure 8: The diphoton mass spectrum, weighted by the ratio of signal-to-background in each category (left) and with background subtracted (right). 
ECAL energy resolution and reconstruction efficiency. Such optimisation process already started: some aspects are being currently finalised and the remaining will be covered and studied during next operation, starting with run 2 in 2015.

During run 1 ECAL collected $1 \%$ of the total expected luminosity, experiencing collisions up to $\sqrt{s}=8 \mathrm{TeV}$ and an average number of events per per bunch crossing $(<$ pile-up $>)$ of 20 . It is designed to operate up to $500 \mathrm{fb}^{-1}$, foreseen by the end of the LHC Phase 1 (end of 2023), when it will have sustained an instantaneous luminosity $\times 2$ higher than the design one, at $\sqrt{s}=$ $13 \mathrm{TeV}$ and $<$ pile-up $>$ up to 60 . In 2025, the High Luminosity LHC will start running at $\sqrt{s}=$ $14 \mathrm{TeV}$, with $<$ pile-up $>\simeq 140$ and $\times 5$ the design instantaneous luminosity. The evolution of the detector conditions and ageing, the very high trigger rate the pile-up mitigation are only some of the challenges foreseen for ECAL in the future.

In order to keep the high level of performance, revised strategies and tunings will be adopted: a calorimeter trigger update, in view of the increased $\sqrt{s}$ to $13 \mathrm{TeV}$ and increased instantaneous lumi, the exploitation of the ECAL timing performance, to help in the pile-up mitigation and particle ID, as well as the improvements in the workflow previously described.

At the end of the LHC Phase 1, the ECAL will be upgraded, in order to cope with the detector ageing and maintain a good level of performance in view of the physics program of the HL-LHC. Up to the current understanding, the EB will be able to withstand the higher pile-up and radiation conditions. However its front-end electronics will be upgraded to move the trigger primitive generation off-detector, allowing more complex trigger algorithms and increased flexibility. Moreover, the ECAL barrel might be cooled to about $8^{\circ} \mathrm{C}$, to decrease the APDs leakage current and the corresponding electronic noise by $\sqrt{2}$. The conditions for the endcaps are foreseen to be acceptable up to $500 \mathrm{fb}^{-1}$, after which the darkening of the crystals and the VPTs response loss will result in an unacceptably low detection efficiency, requiring the replacement of the ECAL EE.

\section{References}

[1] The CMS Collaboration, The CMS Electromagnetic Calorimeter Technical Design Report, CMS TDR 4, CERN/LHCC 97-33 (CERN, Geneva, Switzerland 1997).

[2] Adzic P et al., Energy resolution of the barrel of the CMS Electromagnetic Calorimeter, JINST 2, P04004 (2007)

[3] CMS Collaboration, CMS Physics Technical Design Report, Volume I, CERN-LHCC-2006-021

[4] Adzic P et al., Reconstruction of the signal amplitude of the CMS electromagnetic calorimeter, Eur. Phys. J.C 46, s1 (2006) 23-35

[5] Anfreville M et al., Laser monitoring system for the CMS lead tungstate crystal calorimeter, Nucl. Instr. and Meth. A 594 (2008) 292-320

[6] The CMS Collaboration, The CMS Electromagnetic Calorimeter Technical Design Report, CMS TDR 4, 2.3 Radiation Hardness, 35

[7] CMS Collaboration, Search for a Higgs boson decaying into two photons in the CMS detector, CMS-PAS-HIG-11-021, 2011

[8] CMS Collaboration, Updated measurements of the Higgs boson at $125 \mathrm{GeV}$ in the two photon decay channel, CMS-PAS-HIG-13-001, 2013 